S.-X. Yu

Nagoya Math. J.

Vol. 154 (1999), 103-110

\title{
SOME PROPERTIES OF ISOLATING BLOCKS FOR PLANAR SYSTEMS
}

\author{
SHU-XIANG YU
}

\begin{abstract}
In this paper, some qualitative properties of trajectories inside an isolating block for planar differential equations are obtained.
\end{abstract}

\section{$\S 1$. Introduction}

Consider the differential system defined in the plane

$$
\begin{aligned}
& \frac{d x}{d t}=X(x, y), \\
& \frac{d y}{d t}=Y(x, y) .
\end{aligned}
$$

Suppose $X, Y \in C^{1}$. Let the vector field $V \equiv(X, Y)$ define a flow $f(p, t)$. Let $B \subset R^{2}$ be the closure of a bounded and connected open set with the boundary $\partial B$. In general, $B$ is assumed to be multiply connected. Let $L_{1}, \cdots, L_{n}$ denote its boundary components, where $L_{i} \cap L_{j}=\phi$ for $i \neq j$, and $L_{1}$ the external boundary. Each of them is a smooth simple closed curve. Let int $B$ denote the interior of $B$. We define three subsets $b^{+}, b^{-}$and $\tau$ as follows:

$$
\begin{aligned}
& b^{+}=\{p \in \partial B \mid \exists \varepsilon>0 \quad \text { with } \quad f(p,(-\varepsilon, 0)) \cap B=\phi\}, \\
& b^{-}=\{p \in \partial B \mid \exists \varepsilon>0 \quad \text { with } \quad f(p,(0, \varepsilon)) \cap B=\phi\}, \\
& \tau=\{p \in \partial B \mid V \quad \text { is tangent to } \quad B \text { at } p\} .
\end{aligned}
$$

Definition 1.1. ([1]) If $b^{+} \cap b^{-}=\tau$ and $b^{+} \cup b^{-}=\partial B$, then $B$ is called an isolating block for the flow defined by (1.1).

It follows from the above definition that if $B$ is an isolating block, then all the tangencies to $B$ must be external.

Received July 15, 1997. 
Definition 1.2. Suppose $B \subset R^{2}$ is an isolating block for the flow defined by (1.1). If a trajectory $\Gamma_{1}$ of (1.1) enters $B$ at $M_{1}$ (a strict entrance point) of the external boundary $L_{1}$ of $B$ and then leaves $B$ at $M_{2}$ (a strict exit point) of $L_{1}$ so that int $B$ is divided into two disconnected regions $B_{1}$ and $B_{2}$, then $\Gamma_{1}$ is called a cut trajectory of $B$.

Conley and Easton in [2] have studied generally properties of the isolating block. A special property of planar isolating blocks and an application to the existence of connecting orbits have discussed in [3] (See Lemma 1 of $[3])$. In the present paper, we shall discuss qualitative properties of planar flows inside the isolating block and give some new results.

\section{$\S 2$. The main results}

Suppose $B$ is an isolating block for flow defined by (1.1) and a critical point $Q \in \operatorname{int} B$. Our main aim is to study the existence of elliptic regions of $Q$ (See [4, p.295] for the definition). Therefore, we consider a bounded sectorial region $D$ contained in $B$ with boundary consisting of the critical point $Q$, two semi-trajectory arcs $f\left(M_{1}, R^{+}\right), f\left(M_{2}, R^{-}\right)$and the closed subarc $M_{1} m M_{2}$ of $\partial B$ from $M_{1}$ to $M_{2}$, and such that when $t \rightarrow+\infty$ (or $-\infty), f\left(M_{1}, t\right)$ (or $f\left(M_{2}, t\right)$ ) tends to $Q$, and $M_{1} \in b^{+}, M_{2} \in b^{-}$. Such a sectorial region is said to be adjacent to $\partial B$.

Definition 2.1. Suppose $D$ is a bounded sectorial region adjacent to $\partial B$, as stated above. $D$ is said to be inadmissible if there are a trajectory $\Gamma \subset D$ which tends to $Q$ as $t \rightarrow \pm \infty$ and a circle $\rho$ of radius $r$ small enough with the centre $Q$ such that the interior of each of the curvilinear triangles $Q m_{1} \gamma_{1}$ and $Q m_{2} \gamma_{2}$ is a parabolic sector of $Q([5, \mathrm{p} .164])$ in $\rho$, where it is assumed that $\rho$ intersects $Q M_{1}, \Gamma$ and $Q M_{2}$ at $m_{1}, \gamma_{1}, \gamma_{2}$ and $m_{2}$, respectively (Fig. 1 ).

THEOREM 2.1. Let $B$ be an isolating block for flow defined by (1.1) and a critical point $Q \in$ intB. Let $D$ be an inadmissible sectorial region adjacent to $\partial B$ and let $D$ do not contain any internal boundary components of $\partial B$. Let $D_{1}=D \backslash \bar{G}$, where $G$ is the region enclosed by $\Gamma$ and $Q$. Then there must be at least one critical point of (1.1) in $D_{1}$ (Fig.1). 


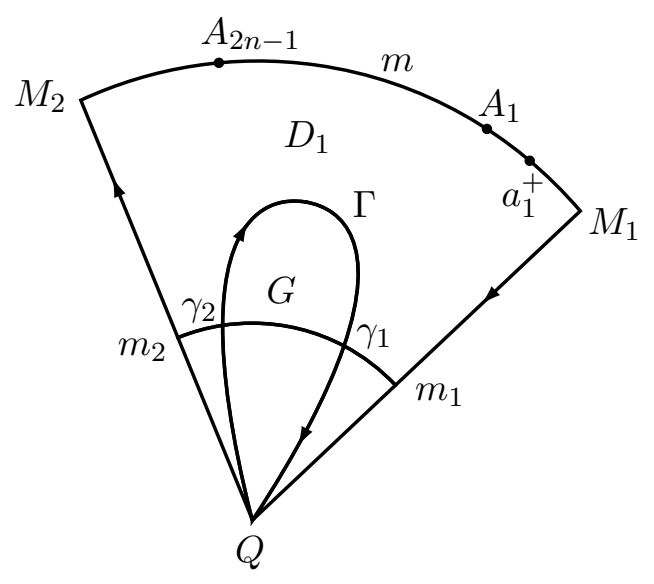

(Figure 1)

Proof. Since $M_{1} \in b^{+}$and $M_{2} \in b^{-}$, there must be at least one tangency to $B$ on the segmental arc $M_{1} m M_{2}$. Let $A_{1}, A_{2}, \cdots, A_{2 n-1}$ be the tangencies to be arranged in numerical order on the arc $M_{1} m M_{2}$. Then the points on the segmental arcs $M_{1} A_{1}, A_{2} A_{3}, \cdots, A_{2 n-2} A_{2 n-1}$ (In what follows, we shall denote these arcs by $1^{+}, 2^{+}, \cdots, n^{+}$respectively) of $M_{1} m M_{2}$ are the strict entrance points of $B$, while the points on the segmental arcs $A_{1} A_{2}, A_{3} A_{4}, \cdots, A_{2 n-1} M_{2}$ (We shall denote them by $1^{-}, 2^{-}, \cdots, n^{-}$respectively) of $M_{1} m M_{2}$ are the strict exit points of $B$ ([5, p.37]). Take arbitrarily an integer $k$ such that $1<k<n$. Consider three consecutive tangencies $A_{2 k}, A_{2 k-1}, A_{2 k-2}$, and four relevant segmental arcs $(k+1)^{+}, k^{-}, k^{+},(k-$ $1)^{-}$. It is easy to see that the positive semi-trajectory originating from every point in a small neighbourhood of $A_{2 k-1}$ on $k^{+}$must leave $D_{1}$ from some point on the segmental arc $k^{-}$, while the positive semi-trajectory originating from every point in a small neighbourhood of $A_{2 k-2}$ on $k^{+}$must leave $D_{1}$ from some point on the segmental arc $(k-1)^{-}$. Therefore, there must be a nonempty set $\beta_{k}^{+} \subset k^{+}$such that for each point $x \in \beta_{k}^{+}$, the positive semi-trajectory $f\left(x, R^{+}\right)$leaves $D_{1}$ neither from a point on $k^{-}$nor from a point on $(k-1)^{-}$for increasing time. Similarly, there must be a nonempty set $\beta_{k}^{-} \subset k^{-}$such that for each point $x \in \beta_{k}^{-}$, the negative semi-trajectory $f\left(x, R^{-}\right)$leaves $D_{1}$ neither from a point on $k^{+}$nor from a point on $(k+1)^{+}$ for all $t<0$. In other words, each of these semi-trajectories can not leave $D_{1}$ from a point on the adjacent segmental arcs. Consider case $k=1$. It is easy to see that if the positive semi-trajectory $f\left(x, R^{+}\right)$originating from a 
point $x \in 1^{+}$tends to $Q$ in $D$ as $t \rightarrow+\infty$, then it must enter the parabolic sector $Q m_{1} \gamma_{1}$ (see Fig.1). So, from the continuity (the solutions depend continuously on initial conditions) it follows that the positive semi-trajectory originating from every point in a small neighbourhood of $x$ on $1^{+}$also tends to $Q$ in $D$ as $t \rightarrow+\infty$. The same argument implies that the positive semitrajectory through a point $x$ of $M_{1} A_{1}$ sufficiently close to $M_{1}$ must tend to $Q$ in $D$ as $t \rightarrow+\infty$. Thus there is a maximal open segmental arc $M_{1} h$ of $M_{1} A_{1}$ such that for every point $x \in M_{1} h$, the positive semi-trajectory $f\left(x, R^{+}\right)$tends to $Q$ in $D$ as $t \rightarrow+\infty$, while the positive semi-trajectory $f\left(h, R^{+}\right)$does not tend to $Q$ in $D$ as $t \rightarrow+\infty$. On the other hand, the same argument used in case $1<k<n$ implies that the positive semi-trajectory originating from every point in a small neighbourhood of $A_{1}$ on $1^{+}$must leave $D_{1}$ from some point on the segmental arc $1^{-}$. Moreover, the set of such points is an open set on the segmental arc $M_{1} A_{1}$. This implies that $h \neq A_{1}$, and the positive semi-trajectory $f\left(h, R^{+}\right)$can neither tend to $Q$ in $D$ nor leave $D_{1}$ from a point on $1^{-}$for increasing time. Hence, for $k=1$, we have proved that there is a nonempty set $\beta_{1}^{+} \subset 1^{+}$such that for each point $x \in \beta_{1}^{+}$, the positive semi-trajectory $f\left(x, R^{+}\right)$can neither tend to $Q$ in $D$ nor leave $D_{1}$ from a point on $1^{-}$for increasing time. For $k=n$, a similar conclusion holds.

Choose arbitrarily $n$ points $a_{i}^{+} \in \beta_{i}^{+}(i=1,2, \cdots, n)$. We now can prove that there is at least one among the positive semi-trajectories $\left\{f\left(a_{i}^{+}, R^{+}\right) \mid i\right.$ $=1,2, \cdots, n\}$ such that it stays in $D_{1}$ for all $t>0$ and does not tend to $Q$ as $t \rightarrow+\infty$. The following proof proceeds by reduction to absurdity. Suppose that each of the semi-trajectories $\left\{f\left(a_{i}^{+}, R^{+}\right) \mid i=1,2, \cdots, n\right\}$ either leaves $D_{1}$ from some point on the segmental arc $M_{1} m M_{2}$ for increasing time or tends to $Q$ as $t \rightarrow+\infty$. Thus, since the positive semi-trajectory $f\left(a_{1}^{+}, R^{+}\right)$can not tend to $Q$ (note $\left.a_{1}^{+} \in \beta_{1}^{+}\right)$, it must leave $D_{1}$ from a point on $M_{1} m M_{2}$ for increasing time. Let it leave $D_{1}$ from some point on $k^{-}$, where $1<k \leq n$. But this means that each of $\left\{f\left(a_{i}^{+}, R^{+}\right) \mid i=2, \cdots, k\right\}$ can not tend to $Q$ as $t \rightarrow+\infty$ for, otherwise it must meet $f\left(a_{1}^{+}, R^{+}\right)$at a point for increasing time and which contradicts uniqueness of solutions. Therefore, each of $\left\{f\left(a_{i}^{+}, R^{+}\right) \mid i=2, \cdots, k\right\}$ must leave $D_{1}$ from a point on $M_{1} m M_{2}$ for increasing time. However, we note that the semi-trajectory $f\left(a_{2}^{+}, R^{+}\right)$can not leave $D_{1}$ from a point on $1^{-}$or $2^{-}$because $a_{2}^{+} \in \beta_{2}^{+}$, hence, it can only leave $D_{1}$ from a point on $i^{-}(i \geq 3)$. This implies that the positive semi-trajectory $f\left(a_{3}^{+}, R^{+}\right)$can not leave $D_{1}$ from a point on $1^{-}$for increasing time for, otherwise it must meet $f\left(a_{2}^{+}, R^{+}\right)$and which 
contradicts uniqueness of solutions, hence, it can only leave $D_{1}$ from a point on $i^{-}(i \geq 4)$. Further, this also implies that the semi-trajectory $f\left(a_{4}^{+}, R^{+}\right)$ can not leave $D_{1}$ from a point on $2^{-}$, hence it can only leave $D_{1}$ from a point on $i^{-}(i \geq 5)$ for increasing time. Repeating an argument used above, one implies that the semi-trajectory $f\left(a_{i}^{+}, R^{+}\right)$can only leave $D_{1}$ from a point on $j^{-}(k \geq j \geq i+1)$ (i.e., on the segmental arc with greater subscript). From this, it follows that the semi-trajectory $f\left(a_{k}^{+}, R^{+}\right)$can not leave $D_{1}$ from a point on $M_{1} m M_{2}$ for increasing time. Moreover, as stated above, it can not also tend to $Q$ as $t \rightarrow+\infty$, therefore, this contradicts the preceding hypothesis. Thus, there must be a positive semi-trajectory $\gamma^{+}$such that it can neither tend to $Q$ nor leave $D_{1}$ from a point on $M_{1} m M_{2}$ for increasing time. By the Poincaré-Bendixson theory of planar systems, the $\omega$-limit set of $\gamma^{+}$must contain critical points or closed orbits. Further, since a closed orbit contains at least one critical point of (1.1) in its interior, this implies that there must be at least one critical point of (1.1) in $D_{1}$. Hence Theorem 2.1 is proved.

COROLlary 1. If the sectorial region $D$ in Theorem 2.1 contains the internal boundary components $L_{i_{1}}, \cdots, L_{i_{k}}$ of $\partial B$, then the conclusion of Theorem 2.1 still holds provided we set $D_{1}=D \backslash\left(\bar{G} \cup \bar{G}_{i_{1}} \cup \cdots \cup \bar{G}_{i_{k}}\right)$, where $G_{i_{1}}, \cdots, G_{i_{k}}$ are the regions enclosed by $L_{i_{1}}, \cdots, L_{i_{k}}$ respectively.

Proof of Corollary 1. We know from the proof of Theorem 2.1 that there is at least one among the positive semi-trajectories $\left\{f\left(a_{i}^{+}, R^{+}\right) \mid i=\right.$ $1,2, \cdots, n\}$, say $f\left(a_{j}^{+}, R^{+}\right)$, such that it stays in $D \backslash \bar{G}$ for all $t>0$ and does not tend to $Q$ as $t \rightarrow+\infty$, where $a_{j}^{+} \in \beta_{j}^{+} \subset j^{+}, A_{2 j-1}$ and $A_{2 j-2}$ are two tangencies close to $a_{j}^{+}$.

Suppose $f\left(a_{j}^{+}, R^{+}\right)$intersects $L_{i_{0}}$ at $b_{j}$, where $L_{i_{0}}$ is one of the internal boundary components $\left\{L_{i_{1}}, \cdots, L_{i_{k}}\right\}$ and $b_{j}$ is a strict exit point of $B$. Consider the segmental arc $A_{2 j-2} a_{j}^{+} A_{2 j-1}$ and its segmental subarc $a_{j}^{+} A_{2 j-1}$. Let $\tilde{a}=\left\{x \in A_{2 j-2} a_{j}^{+} A_{2 j-1} \mid\right.$ the point where $f\left(x, R^{+}\right)$intersects $L_{i_{0}}$ is a strict exit point of $B\}$. From the theorem of continuity (the solutions depend continuously on initial conditions) it follows that $\tilde{a}$ is an open set on the segmental arc $A_{2 j-2} a_{j}^{+} A_{2 j-1}$. Since $A_{2 j-1}$ is a tangency to $B$, the positive semi-trajectory originating from every point in a small neighbourhood of $A_{2 j-1}$ on $a_{j}^{+} A_{2 j-1}$ must leave $B$ from some point on the segmental arc $M_{1} m M_{2}$. Hence there must be at least one boundary point of the set $\tilde{a}$ on $a_{j}^{+} A_{2 j-1}$. Let $a_{0}$ be a boundary point close to $a_{j}^{+}$. Then either there is a 
point $p \in f\left(a_{0}, R^{+}\right)$such that $p$ is a tangency to $L_{i_{0}}$ or $f\left(a_{0}, R^{+}\right)$tends to a critical point of (1.1) in $D_{1}$ as $t \rightarrow+\infty$. In the former case, it follows that there is an internal tangency to $B$. But this is impossible because $B$ is an isolating block. In the latter case, it follows that Corollary 1 holds.

If $f\left(a_{j}^{+}, R^{+}\right)$does not meet any one of the internal boundary components $\left\{L_{i_{1}}, \cdots, L_{i_{k}}\right\}$, then by the Poincaré-Bendixson theory of planar systems it follows that there must be at least one critical point of (1.1) in $D_{1}$, where $D_{1}=D \backslash\left(\bar{G} \cup \bar{G}_{i_{1}} \cup \cdots \cup \bar{G}_{i_{k}}\right)$. Corollary 1 is proved.

Remark 1. Suppose $Q$ is a unique critical point of (1.1) in $B$. Then, Theorem 2.1 means that the fact that there are no internal tangencies to $B$ can imply that there are no certain type of elliptic regions of the critical point $Q$.

Using exactly the same argument used in the proof of Theorem 2.1, we can prove the following theorem. We suppose that the symbols $a_{i}^{ \pm}, i^{ \pm}, \beta_{i}^{ \pm}$ have the same meanings as in Theorem 2.1.

THEOREM 2.2. Let $B$ be an isolating block for flow defined by (1.1). Let $B_{1}$ be the region enclosed by the trajectory arc $M_{1} M_{2}$ of the cut trajectory $\Gamma_{1}$ of $B$ and the segmental arc $M_{1} m M_{2}$ of the external boundary $L_{1}$ of $B$ (Fig.2). Let $A_{1}, A_{2}, \cdots, A_{2 n-1}$ be the tangencies to be arranged in numerical order on the arc $M_{1} m M_{2}$. If $n \geq 2$, then there must be a point $a_{i}^{+} \in i^{+}$and a point $a_{i}^{-} \in i^{-}$such that the semi-trajectories $f\left(a_{i}^{+}, R^{+}\right)$and $f\left(a_{i}^{-}, R^{-}\right)$ stay in $B_{1}$ for all $t>0$ and $t<0$ respectively $(i=1,2, \cdots, n)$.

Proof. First we note, by Definition 1.2, it follows that the positive semi-trajectory originating from every point in a small neighbourhood of $M_{1}$ on $1^{+}$must leave $B_{1}$ from a point on $n^{-}$for increasing time. For $k=n$, a similar conclusion holds. Thus, one can consider $1^{+}$and $n^{-}$as two adjacent segmental arcs.

We proceed by induction. First suppose $n=2$. That is, there are three tangencies $A_{1}, A_{2}, A_{3}$ on the arc $M_{1} m M_{2}$. It is easy to see that the positive (or negative) semi-trajectory originating from any point on $\beta_{i}^{+}$(or $\beta_{i}^{-}$) $(i=1,2)$ stays in $B_{1}$ for all $t>0$ (or $t<0$ ). So, when $n=2$, Theorem 2.2 holds.

Let $k>2$ be an arbitrary positive integer. Let us now make the inductional hypothesis that Theorem 2.2 is true for $2 \leq n \leq k-1$ (i.e., for all those odd numbers which are not greater than $2 k-3)$. We need to show that it is also true for $n=k$ (i.e., for the odd number $2 k-1$ ). 
In fact, from $n=k-1$ to $n=k$, two tangencies $A_{2 k-2}$ and $A_{2 k-1}$ are added to the arc $M_{1} m M_{2}$. The following proof proceeds by reduction to absurdity. Suppose that there is some segmental arc $j^{+}$such that for every point $x \in j^{+}$, the positive semi-trajectory $f\left(x, R^{+}\right)$leaves $B_{1}$ from a point on $M_{1} m M_{2}$ for increasing time. Take arbitrarily a point $a_{j}^{+} \in \beta_{j}^{+}$, then, the positive semi-trajectory $f\left(a_{j}^{+}, R^{+}\right)$must leave $B_{1}$ from the point $a_{l}^{\prime}$ on $l^{-}$for increasing time. The trajectory arc $a_{j}^{+} a_{l}^{\prime}$ divides the segmental $\operatorname{arc} M_{1} m M_{2}$ into three segmental arcs: The segmental $\operatorname{arcs} a_{j}^{+} a_{l}^{\prime}, M_{1} a_{j}^{+}$and $M_{2} a_{l}^{\prime}$ (Fig.2). From the fact that the semi-trajectory $f\left(a_{j}^{+}, R^{+}\right)$leaves $B_{1}$ neither from a point on the adjacent segmental arcs nor from a point on any entrance segmental arc $i^{+}$for increasing time, it follows that there are at least three tangencies on the segmental arc $a_{j}^{+} a_{l}^{\prime}$ of $M_{1} m M_{2}$, while the amount of tangencies on the $\operatorname{arcs} M_{1} a_{j}^{+}$and $M_{2} a_{l}^{\prime}$ of $M_{1} m M_{2}$ is not less than 2 . Thus the number of tangencies on the $\operatorname{arc} a_{j}^{+} a_{l}^{\prime}$ of $M_{1} m M_{2}$ is not greater than $2 k-1-2=2 k-3$. Furthermore, since $a_{j}^{+}$is a strict entrance point of $B_{1}$ and $a_{l}^{\prime}$ is a strict exit point of $B_{1}$, the trajectory arc $a_{j}^{+} a_{l}^{\prime}$ possesses the same property as the $\operatorname{arc} M_{1} M_{2}$ of $\Gamma_{1}$. By the inductional hypothesis it follows that there must be a point $q$ on the arc $a_{j}^{+} A_{2 j-1}$ of $M_{1} m M_{2}$ such that the positive semi-trajectory $f\left(q, R^{+}\right)$stays for all $t>0$ in the region enclosed by the segmental arc $a_{j}^{+} a_{l}^{\prime}$ of $M_{1} m M_{2}$ and the trajectory arc $a_{j}^{+} a_{l}^{\prime}$, hence in $B_{1}$. But since $a_{j}^{+} A_{2 j-1} \subset j^{+}$, this contradicts the preceding hypothesis. Hence we have proved that for each $i \in\{1,2, \cdots, n\}$, there must be a point $a_{i}^{+} \in i^{+}$such that $f\left(a_{i}^{+}, R^{+}\right)$stays in $B_{1}$ for all $t>0$. Similarly, we can prove that for each $i \in\{1,2, \cdots, n\}$, there must be a point $a_{i}^{-} \in i^{-}$such that $f\left(a_{i}^{-}, R^{-}\right)$stays in $B_{1}$ for all $t<0$. Thus Theorem 2.2 is proved.

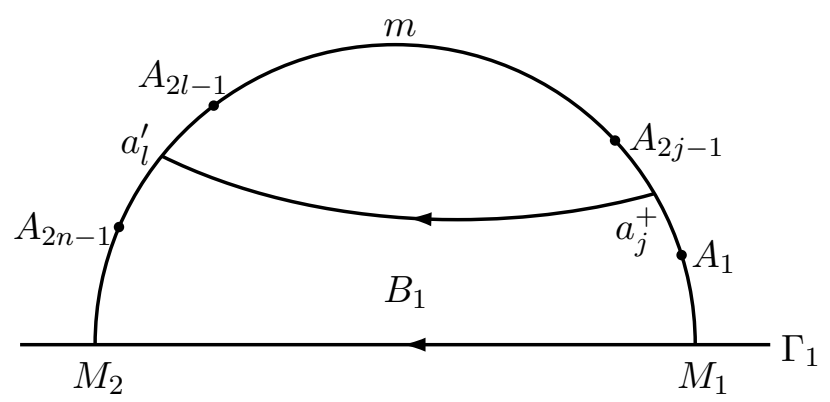

(Figure 2) 


\section{REFERENCES}

[1] C. C. Conley and J. A. Smoller, Topological methods in the theory of shock waves, Proc. Sympos. Pure Math. 23, Amer. Math. Soc., Providence, R. I. (1973), 293-302..

[2] C. Conley and R. Easton, Isolated invariant sets and isolating blocks, Trans. Amer. Math. Soc., 158 (1971), 35-61.

[3] Yu Shu-Xiang, The existence of trajectories joining critical points, J. Differential Equations, 66:2 (1987), 230-242.

[4] A. A. Andronov, E. A. Leontovich, I. I. Gordon, and A. G. Maier, Qualitative Theory of Second-Order Dynamic Systems, New York, Wiley, 1973.

[5] P. Hartman, Ordinary Differential Equations, Birkhauser, Boston, 1982.

Institute of Mathematics

Academia Sinica

100080 Beijing

China

ysx@math03.math.ac.cn 